\title{
On the Geometry of Field Extensions
}

\author{
HANS HAVLICEK
}

Summary. We investigate the spread arising from a field extension and its chains. The major tool in this paper is the concept of transversal lines of a chain which is closely related with the Cartan-Brauer-Hua theorem. Provided that one chain has a "sufficiently large" number of such lines, both this chain as well as the given spread permit a simple geometric description by means of collineations.

0. Every field extension $L$ over $K$ gives rise to a spread together with a system of subsets called chains. Provided that $K$ is in the centre of $L$ these spreads and chains were investigated thoroughly within the wider concept of chain geometries: It is well known that through every point of a subspace belonging to a chain there goes a transversal line of this chain. So every chain is a Segre-manifold (regulus). See [5] for a survey of this topic.

In the present paper we investigate how things will alter when $K$ is not necessarily a part of the centre of $L$.

1. For any vector space $V$ over a (not necessarily commutative) field $K$, denote by $\mathcal{P}_{K}(V)$ the projective space on $V$. The same notation will be used for any subspace of $V$.

Let $L$ be a field. The projective line over $L$ is given by $\mathcal{P}_{L}(L \oplus L)=: \mathcal{P}_{L}$. If $K \neq L$ is a subfield of $L$, then the chains of $\mathcal{P}_{L}$ (with respect to $K$ ) are the images of the standard chain

$$
\left\{\left(k_{0}, k_{1}\right) L \mid(0,0) \neq\left(k_{0}, k_{1}\right) \in K \times K\right\}
$$

under the projective group PGL $\left(\mathcal{P}_{L}\right)$. Cf. [1,320].

Regarding $L \oplus L$ as a right vector space over $K$ yields the projective space $\mathcal{P}_{K}(L \oplus L)=: \mathcal{P}_{K}$. Every point $\left(l_{0}, l_{1}\right) L \in \mathcal{P}_{L}$ gives rise to the subspace $\mathcal{P}_{K}\left(\left(l_{0}, l_{1}\right) L\right)$ of $\mathcal{P}_{K}$. All such subspaces form a spread $\mathbf{S}$ of $\mathcal{P}_{K}$. We shall write $\mathcal{U}:=\mathcal{P}_{K}((1,0) L), \quad V=\mathcal{P}_{K}((1,1) L), \quad W:=\mathcal{P}_{K}((0,1) L)$. Every chain of $\mathcal{P}_{L}$ gives rise to a subset of $\mathbf{S}$ which will be called a chain likewise.

2. Every projectivity $\pi$ of $\mathcal{P}_{L}$ is induced by an $L$-linear $f$ map of $L \oplus L$. But $f$ is also $K$-linear, so that $\operatorname{PGL}\left(\mathcal{P}_{L}\right)$ corresponds to a group of automorphic 
projective collineations of $\mathbf{S}$ which operates 3-fold transitively on $\mathbf{S}$ and hence transitively on the set $\mathbf{C}$ of all chains in $\mathbf{S}$. Therefore it is sufficient to discuss the geometrical properties of the standard chain c, say.

A line $\ell$ of $\mathcal{P}_{K}$ is called transversal line of a chain $\mathbf{k}$, if $y \in \mathbf{k} \mapsto \ln y$ defines a bijection of $\mathbf{k}$ onto $\ell$.

THEOREM 1. There exists a transversal line of the standard chain $\mathrm{c}$ passing through $(a, 0) K \in U$ if, and only if, $a^{-1} K a=K$.

Proof. Let $\ell$ be a line which intersects $u, v$ and $W$. Then $\ell$ contains $(a, 0) K \in U,\left(a^{\prime}, a^{\prime}\right) K \in V,\left(0, a^{\prime \prime}\right) K \in W$, say, with $a, a^{\prime}, a^{\prime \prime} \in L^{\times}:=L \backslash\{0\}$. By collinearity of these points $a=a^{\prime}=a^{\prime \prime}$.

Now take any element $y=\mathcal{P}_{K}((1, y) L) \in \mathrm{c}$ with $\mathrm{y} \in K^{\times}$. We deduce that $\ell$ has a point in common with $y$ if, and only if, there exist skalars $x_{0}, x_{1} \in K^{\times}$, $b \in L^{\times}$such that

$b=a x_{0}, y b=a x_{1}$.

As $x_{0}, x_{1}$ are right homogeneous coordinates, we may put $x_{0}:=1$, whence $a=b$ and $a^{-1} y a \in K$. So $\ell$ intersects all elements of $\mathrm{c}$ if, and only if, $a^{-1} K a \subset K$.

In the same manner as above $K \subset a^{-1} K a$ can be shown to be necessary and sufficient that every point of $\ell$ lies in at least one element of $\mathbf{c}$

Let $a \in L^{\times}$and $a^{-1} K a=K$. The restriction of the inner automorphism

$\psi_{a}: L \rightarrow L, x \mapsto a^{-1} x a$

to $K$ induces an automorphism $\varphi_{a}$ of $K$. If $\varphi_{a}$ is inner, then $a^{-1} y a=u^{-1} y u$ for all $y \in K$ and some $u \in K^{\times}$, whence $a \in Z_{L}(K)^{\times} \cdot K^{\times}$, where $Z_{L}(K)$ denotes the centralizer of $K$ in $L$. Conversely every $a \in Z_{L}(K)^{\times} \cdot K^{\times}$gives rise to an inner automorphism of $K$.

Let $a \in K^{\times}$and $a^{-1} K a \subset K$. Then $\psi_{a}$ restricted to $K$ is an isomorphism $\varphi_{a}$ of $K$ onto a subfield of $K$. Clearly, $\varphi_{a}$ is linear when $K$ is regarded as a right vector space over $f \operatorname{ix}\left(\varphi_{a}\right):=\left\{y \in K \mid \varphi_{a}(y)=y\right\}$. Provided that the right degree of $K$ over $\operatorname{fix}\left(\varphi_{a}\right)$ is finite, $\varphi_{a}$ turns out to be surjective or, in other words, an automorphism of $K$. Consequently such an $a \in K^{\times}$yields a transversal line of c.

One special case is worth noting: If the centre $Z_{L}(L)=: Z$ of $L$ is a subfield of $K$ and $[K: Z]$ is finite then, by a theorem of Skolem and Noether (cf. e.g. [2,46]), every automorphism of $K$ which fixes $Z$ elementwise extends to an inner automorphism of $L$ and hence gives rise to a transversal line of $\mathbf{c}$.

3. We investigate the set of all transversal lines of the standard chain $\mathbf{c}$. Obviously $k:=(1,0) K \vee(0,1) K$ is a transversal line of c. It will be called the standard transversal line of $c$. If $\ell$ is any transversal line of $c$ then 


$$
\alpha: k \rightarrow \ell, k \cap Y \mapsto \ln Y \text { (with } \mathcal{Y} \in \mathrm{c} \text { ) }
$$

is a well defined bijection of $k$ onto $\ell$. Suppose that $\ell$ carries the point $(a, 0) K$. Then this $\alpha$ is given explicitly by

$$
(1, y) K \mapsto(a, y a) K=\left(a, a\left(a^{-1} y a\right)\right) K,
$$

whence $a \in Z_{L}(K)^{\times} \cdot K^{\times}$characterizes $\alpha$ as being a projectivity. If $\alpha$ is a projectivity, then we shall say that $k$ and $\ell$ are projectively linked transversal lines. This is an equivalence relation on the set of transversal lines of $\mathbf{c}$.

THEOREM 2. Suppose that $\ell_{i}(i \in I)$ are transversal lines of the standard chain c and write $A_{i}:=\ell_{i} \cap \mathcal{U}$.

(a) If $\left\{A_{i} \mid i \in I\right\}$ is an r-frame, then all $\ell_{i}^{\prime}$ 's are projectively linked.

(b) If $\ell_{j}$ and $\ell_{k}$ are not projectively linked whenever $j, k \in I$ are different, then $\left\{A_{i} \mid i \in I\right\}$ is an independent set of points and no other transversal line of $\mathbf{c}$ is incident with a point of $\operatorname{span}\left\{A_{i} \mid i \in I\right\}$.

Proof. (a) Let $\left\{A_{i} \mid i \in I\right\}$ be an $r$-frame with $I=\{0, \ldots, r+1\}$, say. Hence for all $\mathcal{Y} \in \mathbf{c}$ the points of intersection $\ell_{0} \cap \mathcal{Y}, \ldots, \ell_{r+1} \cap \mathcal{Y}$ form a frame of an $n$-dimensional subspace of $\mathcal{Y}$. Thus the projection onto the line $\ell_{1}$ with centre $\ell_{2} \vee \ldots \vee \ell_{r+1}$ takes $\ell_{0} \cap \mathcal{Y}$ to $\ell_{1} \cap \mathcal{Y}$. The same argument holds for any two different lines $\ell_{i}$ and $\ell_{j}$.

(b) Suppose that $\left\{A_{i} \mid i \in I\right\}$ is dependent. Then $\left\{A_{i} \mid i \in I^{\prime}\right\}$ for some finite subset $I^{\prime} \subset I$ is a frame of an $r$-dimensional subspace of $U$ with $r \geq 1$. Thus two different transversal lines are projectively linked by (a), an absurdity.

THEOREM 3. Denote by $\mathcal{T}$ the set of all points of $U$ which are incident with a transversal line of $\mathrm{c}$ which is projectively linked with the standard transversal line $k$. With $\mathcal{T}$ being regarded as a trace space of $U$, the projective space on the right vector space $Z_{L}(K)$ over the centre of $K$ is isomorphic to $\mathcal{T}$. Moreover independence of points with respect to the trace space $\mathcal{T}$ is equivalent to independence with respect to $U$.

Proof. A bijection $\iota$ of the projective space on $Z_{L}(K)$ over $Z_{K}(K)$ onto $\mathscr{T}$ is given by

$$
a Z_{K}(K) \mapsto(a, 0) K \quad(a \neq 0) .
$$

Assume that for $a \in Z_{L}(K)$ there exist different elements $a_{1}, \ldots, a_{n} \in Z_{L}(K)$ which are linearly independent over $K$ such that

$$
a=a_{1} x_{1}+\ldots+a_{n} x_{n} \text { with } x_{i} \in K^{\times} \text {. }
$$

We read off from

$$
\text { ay }=\sum a_{i}\left(x_{i} y\right)=y a=\sum y a_{i} x_{i}=\sum a_{i}\left(y x_{i}\right) \text { for all } y \in K,
$$

that all $x_{i}^{\prime}$ s are in the centre of $K$. On the other hand any linear combination 
$\sum_{i} a_{i} x_{i} \quad$ with $a_{i} \in Z_{L}(K)^{\times}, x_{i} \in Z_{K}(K)$

belongs to the centralizer of $K$ in $L$.

Thus $\iota$ is collineation of the projective space on $Z_{L}(K)$ onto the trace space $\mathfrak{T}$ and independence with respect to $\mathcal{T}$ and $U$ is equivalent.

Now suppose that transversal lines $k$ and $\ell$ are not projectively linked. By theorem 2 the pedal point $(b, 0) K$ of $\ell$ in $U$ does not belong to span $\mathcal{T} \subset U$. The standard chain $\mathbf{c}$ is elementwise invariant under the collineation

$$
\mu_{b}: \mathcal{P}_{K} \rightarrow \mathcal{P}_{K},\left(l_{0}, l_{1}\right) K \mapsto\left(l_{0} b, l_{1} b\right) K
$$

and the transversal lines of $\mathbf{c}$ are permuted bijectively. The relation "projectively linked" is being preserved under $\mu_{b}$ as well as $\mu_{b}{ }^{-1}$. Since $\mu_{b}(k)=\ell$, all results established for $k$ carry over to $\ell$. As an immediate consequence of theorems 2 and 3 we state:

THEOREM 4. Let $\ell_{i}(i \in I)$ be a family of transversal lines of the standard chain c. Suppose that $\ell_{j}$ and $\ell_{k}$ are not projectively linked whenever $j, k \in I$ are different. Denote by $\mathcal{T}_{i} \subset U$ the set of all points which are incident with a transversal line of $\mathrm{c}$ that is projectively linked with $\ell_{i}$. Then $\left\{\operatorname{span\mathcal {T}_{i}|i\in I\} }\right.$ is an independent set of isomorphic subspaces of $U$.

4. As an application of the previous results here is a simple geometric proof of the Cartan-Brauer-Hua theorem (cf. e.g. $[1,323]$ ):

Suppose that $K \neq L$ and $a^{-1} K a=K$ for all $a \in L^{\times}$. Then every point of $U$ is on a transversal line of $\mathbf{c}$. By theorem 2 all transversal lines of $\mathbf{c}$ are projectively linked with $k$, whence $\varphi_{a} \in \operatorname{Aut}(K), y \mapsto a^{-1}$ ya is inner. We deduce from theorem 3 and $\mathcal{T}=U$ that $K$ is isomorphic to $Z_{K}(K)$. Therefore $K$ is commutative and $\varphi_{a}=\mathrm{id}_{K}$. Thus $K$ lies in the centre of $L$, as required.

5. Denote by $\tilde{A}$ the join of $W$ with any point $(a, 0) K \in U$ and put $\mathscr{A}:=\tilde{A} \mathbb{W}$. We define a map

$$
\rho: \mathbf{S} \backslash\{W\} \rightarrow \mathcal{A}, x \mapsto \operatorname{An} X
$$

In algebraic terms we have $\mathcal{P}_{K}\left(\left(l_{0}, l_{1}\right) L\right) \mapsto\left(a, l_{1} l_{0}^{-1} a\right) K$, whence $\rho$ is a bijection. Note that $\mathcal{A}$ is an affine space whose parallelism is given by $W$ as hyperplane at infinity. Obviously $\mathcal{A}$ is isomorphic to the affine space on the vector space $L$ over $K$. Those chains through $W$ which have a transversal line in $\tilde{A}$ are in one-one correspondence with the lines of $\mathcal{A}$.

THEOREM 5. Let $a_{0}=1, a_{1} \in L^{\times}$and write $A_{0}, A_{1}$ for the affine spaces given by $\left(W \vee\left(0, a_{0}\right) K\right) \backslash W,\left(W \vee\left(0, a_{1}\right) K\right) \backslash W$, respectively. The bijection 


$$
\beta_{01}: A_{0} \rightarrow A_{1}, X \cap A_{0} \mapsto X \cap A_{1} \quad(X \in S \backslash\{W\})
$$

is an affinity if, and only if, $a_{1}{ }^{-1} K a_{1}=K$.

Proof. (a) If $\beta_{01}$ is an affinity, then $\beta_{01}$ extends to a collineation $\kappa_{01}: \tilde{A}_{0} \rightarrow \tilde{A}_{1}$, say, which takes the standard transversal line $k$ of $\mathrm{c}$ to a transversal line of $\mathrm{c}$ passing through $\left(a_{1}, 0\right) K$. We obtain $a_{1}{ }^{-1} K a_{1}=K$ by theorem 1.

(b) Suppose that $a_{1}{ }^{-1} K a_{1}=K$. The bi jection $\beta_{01}$ maps $(1, l) K \in \mathscr{A}_{0}$ to $(a, l a) K$. But $l(\in L) \mapsto l a_{1}(\in L)$ is $K$-semilinear, so $\beta_{01}$ is affine.

Clearly the affine structure of $\mathcal{A}$ can be re-transferred to $\mathbf{S} \backslash\{\boldsymbol{W}\}$. This gives a residual affine space of $(\mathbf{S}, \mathbf{C})$. We read off from theorem 5 that this affine structure on $\mathbf{S} \backslash\{\mathcal{W}\}$ is uniquely determined by $\mathcal{W}$ together with one chain $\mathbf{k}$ through $W$ such that $\mathbf{k} \backslash\{\mathcal{W}\}$ is an affine line.

When $K$ is commutative and the right degree of $L$ over $K$ is finite, say $r+1$, then a point model of (S,C) may be found on a Grassmannian manifold. The map $\rho$ can be extended to all $r$-dimensional subspaces of $\mathcal{P}_{K}$ whose intersection with $\tilde{\mathcal{A}}$ is precisely one point. Up to a projective collineation this extension of $\rho$ equals the product of the Grassmann map $\gamma$ with a suitable projection of the Grassmannian. The restriction of this projection to $\gamma(\mathbf{S})$ is "stereographic", since $\gamma(W)$ is the only point of $\gamma(\mathbf{S})$ without image. So the situation is similar to ordinary chain geometry; cf. [5, chapter 18.6.4].

THEOREM 6. Suppose that there exists a basis $\left\{\left(a_{i}, 0\right) K \mid i \in I\right\}$ of $u$ with transversal lines $\ell_{i}$ of the standard chain $\mathrm{c}$ passing through these points, respectively. Let $0 \in I, a_{0}=1$ and write $\tilde{A}_{i}:=W v\left(a_{i}, 0\right) K$. Then there exist collineations $\kappa_{0 i}: \tilde{A}_{0} \rightarrow \tilde{A}_{i}$ such that

$$
X=\operatorname{span}\left\{\kappa_{0 i}\left(X \cap A_{0}\right) \mid i \in I\right\} \text { for all } X \in \mathrm{c} .
$$

Proof. Define $\kappa_{0 i}$ as the extension of the affinity $\beta_{0 i}$ according to the proof of theorem 5. Then $X=\operatorname{span}\left\{\kappa_{0 i}\left(X \cap A_{0}\right) \mid i \in I\right\}$, since $\mathbf{S}$ is a spread.

Generalizing a terminology introduced in [4] we may say that the spread $\mathbf{S}$ is generated by the family $\kappa_{0 i}$ of collineations. (Cf. [6,pp.299] for a similar, but nevertheless different result on pappian spreads.) If we restrict the collineations $\kappa_{0 i}$ to the standard transversal line $k$, then we obtain a geometric description of the standard chain c. By transformation under automorphic collineations of $\mathbf{S}$ this description carries over to any chain of the spread S. Provided that $K$ is in the centre of $L$, the conditions of theorem 6 are automatic and we have re-established the result that chains are Segremanifolds. 
It is easy to give examples of fields $K, L$ such that the conditions of theorem 6 are met. Assume that $L$ is a field of quaternions over a commutative field $P$ with $\{1, \mathrm{i}, \mathrm{j}, \mathrm{k}\}$ denoting the usual basis of $L$ over $P$.

1. Let $P=\mathbb{R}$ and $K=\mathbb{R}(i)$ a subfield of complex numbers. Putting $a_{0}=1$, $a_{1}=\mathrm{j}$ shows that $\mathbf{S}$ is a spread generated by a non-projective collineation. Cf. [4].

2. Let $P=\mathbb{Q}(\sqrt{2}\urcorner$ ) and $K=\mathbb{Q}(\mathrm{i})$. Put $\left.a_{0}:=1, \quad a_{1}:=\sqrt{2}, \quad a_{2}:=\mathrm{j}, \quad a_{3}:=\mathrm{j} \sqrt{2}\right\urcorner$. Here $\kappa_{01}$ is projective while $\kappa_{02}$ and $\kappa_{03}$ are non-projective collineations.

3. Let $P=\mathbb{Q}(\sqrt{2})$ and $K=\mathbb{Q}(\mathrm{i}, \mathrm{j}, \mathrm{k})$, viz. the quaternions over $\mathbb{Q}$. Put $a_{0}:=1$, $a_{1}:=\sqrt{2}$. Thus $\kappa_{01}$ is a projective collineation, every chain is a regulus in the sense of B. Segre [7,319] and $\mathbf{S}$ is an elliptic linear congruence of lines according to a definition given in [3].

In this last example we are already "very close" to the description of the spread $\mathbf{S}$ and its chains when $K$ is a subfield of the centre of $L$.

\title{
REFERENCES
}

[1] Benz, W., Vorlesungen über Geometrie der Algebren, Grundlehren Bd. 197, Berlin Heidelberg New York, Springer, 1973.

[2] Cohn, P.M., Skew Field Constructions, Cambridge University Press, Cambridge, 1977.

[3] Havlicek, H., On Sets of Lines Corresponding to Affine Spaces, in, Proceedings of "Combinatorics '88" (Ravello, Italy), to appear.

[4] Havlicek, H., Dual spreads generated by collineations, Simon Stevin 64 (1990), 339-349.

[5] Herzer, A., Chain Geometries, in: Buekenhout, F. (ed.), Handbook of Incidence Geometry, Dordrecht Boston, D. Reidel, to appear.

[6] LunARdon, G., Fibrazioni planari e sottovarietà algrebraiche della varietà di Grassmann, Geom. Dedicata 16 (1984), 291-313.

[7] Segre, B., Lectures on Modern Geometry, Roma, Ed. Cremonese, 1961.

\author{
Institut für Geometrie \\ Technische Universität \\ Wiedner Hauptstrasse 8-10 \\ A-1040 Wien \\ Austria
}

